Vol. 3, n² | 1999

Varia

\title{
Savoirs féminins et construction de la vérité : les femmes dans la preuve testimoniale en Castille au $\mathrm{XIII}^{\mathrm{e}}$ siècle
}

Marta Madero

\section{CpenEdition \\ Journals}

Édition électronique

URL : https://journals.openedition.org/chs/887

DOI : $10.4000 /$ chs. 887

ISSN : 1663-4837

Éditeur

Librairie Droz

Édition imprimée

Date de publication : 1 juin 1999

Pagination : $5-21$

ISBN : 2-600-00398-3

ISSN : 1422-0857

Référence électronique

Marta Madero, «Savoirs féminins et construction de la vérité : les femmes dans la preuve testimoniale en Castille au XIII siècle », Crime, Histoire \& Sociétés / Crime, History \& Societies [En ligne], Vol. $3, n^{\circ} 2$ I 1999, mis en ligne le 03 avril 2009, consulté le 23 mars 2022. URL : http://journals.openedition.org/ chs/887 ; DOI : https://doi.org/10.4000/chs.887 


\title{
Savoirs féminins et construction de la vérité:
}

\author{
les femmes dans la preuve testimoniale \\ en Castille au XIII siècle
}

\begin{abstract}
Marta Madero'
Au XIII siècle, dans le royaume de Castille, des systèmes normatifs qui pensent différemment le lieu des femmes dans la construction de la vérité judiciaire se succèdent et se croisent. De façon schématique, on pourrait parler de deux modèles d'acceptation et d'exclusion de leur témoignage. Le premier limite la connaissance des femmes aux situations «féminines». Le second fonde les hiérarchies propres au sens sur des logiques qui débordent largement la problématique de l'identité sexuelle. À l'intérieur de ces deux constructions, la vérité dépend, à la fois, de l'idée de vraisemblance, des critères sociaux de validation des récits, de la logique argumentative et de la forme de la perception sensorielle.
\end{abstract}

In the XIIIth-century kingdom of Castille, a succession of normative systems comprising different conceptions of the place of women in the construction of judicial truth appeared and cross paths. Roughly speaking, we could talk about two models of acceptance and rejection of women's testimony. The first restricts women's knowledge to «female» situations. The second bases the hierarchies connected with meaning on logics covering much more than the problematic of sexual identity. Within these two constructions, truth depends simultaneously on the idea of likeliness, the social criteria validating the accounts, on argumentative logic and on the form of sensory perception.

\footnotetext{
A u XIII ${ }^{\mathrm{e}}$ siècle, dans le royaume de Castille, des systèmes normatifs qui pensent différemment le lieu des femmes dans la construction de la vérité judiciaire se succèdent et se croisent. De façon schématique, on pourrait parler de deux modèles d'acceptation et d'exclusion de leur témoignage. Le premier, celui des fors, repris à la fin du siècle par les Leyes del Estilo, limite la connaissance des femmes aux situations «féminines». Le second, celui que manifestent essentiellement les Siete Partidas, fonde les hiérarchies propres au sens sur des logiques qui débordent largement la problématique féminine. A l'intérieur de ces deux constructions, la

1 Marta Madero est professeur à l'Universidad de Buenos Aires (Instituto de Historia Antigua y Medieval) à l'Universidad Nacional de general Sarmiento (Argentine) et à l'Université Paris XIII. Elle a notamment publié: Manos violentas, palabras vedadas. La injuria en Castilla y Léon (siglos XIII-XV), prologue de Jacques Le Goff, Taurus, Madrid, 1992 «L'injure et le corps en Castille aux $\mathrm{XIII}^{e}$ et XIV" siecles ", Atalaya. Revue Française d'Études Hispaniques, 1994, 5 ; «Formas de la justicia en la obra jurídica de Alfonso X el Sabio», Hispania, 1996, 56; «Façons de croire. Les témoins et le juge dans l'œuvre juridique d'Alphonse X Le Sage, roi de Castille », Annales H.S.S., 1999, 1, 197-218. Elle prépare actuellement un ouvrage sur «Les régimes et pratiques de vérité dans la Castille des XII'-XIV ${ }^{e}$ siècles ".
} 
vérité dépend, à la fois, des possibilités d'accès au savoir et des critères de validation des récits.

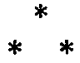

Qu'en est-il du témoignage des femmes dans les Leyes del Estilo ${ }^{2}$ ? Leur titre 96 affirme que leur témoignage ne peut être accepté que dans les causes portant sur des acquisitions ou des ventes, dans les querelles ou les procès pour maléfices qui ne concernent que des femmes. Il affirme aussi, que leurs déclarations sont éventuellement une présomption qui permet de mettre à la torture, à condition qu'aucun récit masculin ne soit en contradiction avec ce qu'elles ont dit. Mais -et c'est l'essentielle témoignage des femmes ne peut être accepté dans des litiges civils ou criminels dans lesquels elles ne sont pas personnellement impliquées. Le texte limite ainsi étroitement la pertinence que les Siete Partidas conféraient au témoignage des femmes, en les acceptant dans tout procès, même les procès criminels, à condition qu'elles fussent de «bonne réputation ${ }^{3}$. Une seule exception était faite : elles ne pouvaient pas être témoin d'un testament, parce que, comme l'explique déjà la Summa codicis attribuée à Irnerius, être témoins des dernières volontés n'est ni un offitium, ni une probatio, mais une sollemnitas ${ }^{4}$.

Les Siete Partidas sont ainsi le texte juridique castillan qui a conféré la plus large crédibilité aux témoignages féminins. De ce point de vue, le texte semble être l'aboutissement d'une évolution, dans la mesure où il étend la legitimité des dépositions féminines à des domaines dans lesquels tous les textes antérieurs les refusaient. L'Espéculo ${ }^{5}$, texte de peu antérieur aux Partidas, leur refusaient le droit de témoigner non seulement dans les testaments, mais encore dans les procès criminels. Dans ce dernier cas, c'est seulement s'il était impossible d'obtenir le témoignage d'un homme que l'on pouvait avoir recours aux femmes dont les dépositions n'étaient toutefois que des simples indices ${ }^{6}$ permettant l'application de la question à ceux dont

2 Les Leyes del Estilo sont un texte de datation difficile puisqu'aucun manuscrit complet ne nous est parvenu. Les différents fragments qui le composent proviennent certainement de moments divers et ne font pas, loin s'en faut, systématiquement référence au Fuero Real, comme le suggère l'édition de los Códigos Españoles Concordados y Comentados, t. I, Madrid, 1850. Le titre 96 s'appuie sans doute sur les Siete Partidas et non pas sur le Fuero Real. C'est au Prof. J.L. Bermejo que je dois des renseignements importants sur la nature de ce texte.

3 Siete Partidas 3,16,17. L'édition utilisée est celle qui contient les gloses de Gregorio Lopez, Salamanque, Andrea de Portonariis, 1555 (fac-simile : Boletín Oficial del Estado, 3 vols., 1974).

4 Ed, par H. Fitting, (Berlin, 1894, chap. De Testibus, pp. 91-92). Dans le droit romain, D 22.5.18 considère que l'incapacité au témoignage touche les femmes adultères et D. 28.1.20.6 interdit leur témoignage dans les testaments. Linterdiction dans les procès criminels est courante. Quant au droit canon, jusqu'au Décret les femmes sont interdites de témoignage, cf. Yves de Chartres, Comp.Pan. VII, 49, PL 161, mais la C. 15 q. 3 c. 2 , qui s'inspirerait du D 22.5.18, accepte le témoignage des femmes, la C.33.q.5.c.17 les exclut des testaments et des causes criminelles, comme le fait l'Espéculo.

5 Espéculo IV.7.3 (éd. R.A. MacDonald, Madison, 1990).

- Le mot indicium, présent dans les sources romaines (C.4.19.25), est utilisé par les légistes, et en particulier par les commentateurs dans le sens de preuves inférieures à la probatio semiplena, (Bartole, Opera, éd. Lyon, 1541, D 27.1.2, Balde, Opera, éd. Lyon, 1543, D.22.3.7 et D 27.1.2 «indicium est minus quam semiplena probatio ") mais cette distinction n'est pas toujours faite, et le mot désigne en général des moyens peu déterminants, soit parce que viciés par une cause spéciale, comme le témoignage de certains sans capacité, soit parce qu'ils gardent peu de rapport avec le factum probandum. Ils servent surtout à permettre la torture: « indicia ad torturam ». Balde les définit aussi comme «pro- 
la culpabilité était insuffisamment prouvée. Contrairement au témoignage des serfs, qui pouvait se trouver ratifié par la torture, celui des femmes ne donne que des preuves nécessairement indirectes.

Peu d'années avant la rédaction de l'Espéculo, le roi Sage avait imposé le Fuero Real à de nombreuses villes. Ce texte, de style très différent de ceux qui viendront ensuite, était encore proche du système foral et par son contenu et par son langage, et il limitait plus radicalement encore le témoignage féminin. «Toute femme voisine, ou fille de voisin peut témoigner dans les choses qui seraient faites ou dites dans les bains, au four ou au moulin, à la rivière ou à la fontaine, ou pendant le filage ou le tissage ou lors des accouchements, ou lors de mariages de femmes, (sic) ou dans d'autres faits féminins, et non pas dans les autres choses, sinon dans celles que cette loi ordonne ${ }^{7}$. En effet, c'est d'abord dans les constats de viol, de virginité et de grossesse que les femmes sont régulièrement appelées à témoigner, et la définition des lieux et pratiques spécifiquement féminins est également courante dans les fors. À cette limitation se substituait ou s'en ajoutait souvent une autre dans ces textes, celle qui prenait comme critère le montant de la demande, les femmes ne pouvant intervenir que dans les litiges inférieurs à une certaine somme.

Ainsi, le Fuero Real, qui au milieu du XIII ${ }^{\mathrm{e}}$ siècle marque une avancée décisive dans le processus de constitution d'un droit unique ${ }^{8}$, et certains titres des Leyes del Estilo, qui dans le dernier tiers du siècle sanctionnent la faillite partielle de cette tentative, demeurent proches de la tradition forale, aussi bien en ce qui concerne la catégorie de «voisin», typique du contexte des concejos, que dans l'affirmation de l'existence de certains espaces et pratiques féminins qui seuls donnent validité au témoignage des femmes.

De là, le premier problème que voudrait aborder cet exposé : comment comprendre le retour en arrière par rapport aux Siete Partidas en ce qui concerne la possible acceptation des témoignages féminins? Pourquoi se trouvent-ils enfermés dans des limites plus étroites après le grand texte du roi Sage?

Résumons d'abord les différentes modalités d'appréciation du témoignage féminin. Premièrement, et cela reste valable dans tous les textes dont il a été question, les femmes doivent être de bonne réputation, ce qui, dans le contexte foral, est généralement lié au statut de vecindad. Deuxièmement, dans certains textes, elles ne peuvent témoigner que dans les «questions des femmes». Troisièmement, dans d'autres cas, leurs témoignages ne sont acceptés que dans des litiges impliquant un montant modeste. Et, finalement, on considère qu'elles ne produisent que des indices, que des traces d'une possible vérité qui devra se manifester dans d'autres récits que les leurs. Ces manières d'évaluer la force et la légitimité du récit féminin, de tenir pour

batio indicativa »: qui donnent au juge des indications pour chercher la vérité. lls differrent donc de la "probatio perspectiva sive intuitiva» par témoins de visu, et de la preuve «praesumptiva» par présomptions probables (C 4.19.25) Cf.Lévy (1939)

7 Fuero Real 2.8.8 «Toda mugier uezina ofiia de uezino pueda testiguar en cosas que fueren fechas o dichas en banno o en forno o en molino o en río o en fuente o sobre filamientos o sobre teximientos o sobre partos o en casamientos de mugier o en otros fechos mugeriles e non en otras cosas sinon en las que manda la ley. Si fuere mugier que ande en semiança de uarón, non queremos que testimonie, sinon en cosa que sea contra rey o contra su sennorío»(éd. G.Martinez Diez, Avila, 1988).

La tentative la plus consistante à donner un droit unique au royaume est sans doute celle d'Alphonse $X$, mais avant lui, il y avait eu d'autres ébauches de ce processus dans la concession réitérée d'un même modèle foral de la part d'Alphonse VIII, et surtout, dans l'imposition du Fuero Juzgo par Ferdinand III, père du roi Sage, aux régions nouvellement reconquises d'Andalousie. 
preuve la connaissance donnée par leur sens, ou d'autoriser les seuls savoirs liés aux pratiques proprement féminines, doivent être compris dans le cadre plus général dans lequel s'élabore la vérité judiciaire.

D'où la seconde intention de ce travail. Au delà de l'immédiateté de l'infériorité féminine, telle que la proclament les discours masculins, ou que la manifeste la condition faite aux femmes, il s'agit pour nous de comprendre la disqualification, ou l'acceptation limitée de leur parole en fonction de la cohérence interne qui organise le système de la preuve judiciaire. Un tel projet, s'il fait son profit de travaux récents menés dans le domaine de l'histoire des femmes, s'en distingue par l'accent mis sur les mécanismes propres au langage juridique.

Le moyen-âge a toujours manifesté une nette préférence pour la preuve testimoniale. Cette préférence, contraire à celle du droit romain classique, fait que les écrits sont moins souvent allégués, qu' on cherche la "voix vive» des témoins au détriment de la «voix morte» des textes ${ }^{9}$. Seuls les témoins, dit Innocent IV dans son commentaire aux Décrétales, sont de droit naturel. Ce n'est que par un artifice du droit, par un miracle du droit positif, que l'on peut donner la peau d'un animal mort comme référent du réel ${ }^{10}$. Certains brefs ordines, comme celui de Jacobo de las Leyes (la Summa de los nove tiempos), ou celui de Arias de Balboa (Los IX tien$p o s)^{11}$, ne font même mention que des témoins dans le titre des preuves; et cette préférence pour les témoins «sensoriels » existait déjà dans de nombreux fors, dont certains étaient marqués par le droit romain renaissant, qui était ignoré par d'autres. Mais la validation des témoignages n'est pas une chose simple, et elle obéit, comme on le verra, à des critères complexes.

Deux observations sont ici indispensables dans la mesure où la disqualification du témoignage féminin, ou l'attribution d'une véridicité limitée aux paroles de femmes, ne suppose pour autant, ni que le savoir masculin soit doté d'une objectivité sans failles, ni qu'il soit possible d'accéder à une vérité qui soit dans une relation de transparence avec la réalité passée. L'idée, introduite par Michel Foucault, des «régimes de vérité», tout comme celle de "formes de vie» empruntée à Wittgenstein ou des «modes d'expérience» développée par Michael Oakeshott rendent compte d'une pluralité ancienne des modes de vérité. Aristote remarquait déjà qu'à toute pratique correspond un degré approprié de certitude, et les juristes du moyenâge élaborent un vérité propre à l'espace judiciaire qui est entièrement déterminée par le probable, l'incomplet, l'absent. C'est pourquoi les deux observations que je voudrais formuler avant d'entreprendre l'analyse du témoignage féminin concernent la notion de vérité et le rôle du juge.

9 L'expression «voix morte» fait référence à la Novelle 73, qui dans son chap. 3 dit «Ea quae viva dicuntur voce et cum iureiurando, haec digniora fide quam scripturam ipsam secundum se subsistere »[nous estimons, en vérité, qu'est plus digne de foi ce qui est dit de vive voix et sous serment que la survivance du document écrit lui-même]. Ce texte est employé au moyen-âge séparé de son contexte qui visait la procédure de vérification des écritures, et ne préférait les tếmoins instrumentaires qu'à la comparaison des écrits. Sur cette question, Lévy (1939, p. 87-95).

10 À propos de X.2.22.14 $\mathrm{n}^{\circ} 1$ (éd. Venise, 1570), où il explique que seul le Pape ou l'Empereur peuvent nommer les tabelliones parce qu'il n'y a qu'eux qui peuvent créer le droit et leur déléguer ainsi ce pouvoir créateur qui leur permettra de faire fonctionner cette fiction du droit qui fait que l'on croit en la peau d'un animal mort (quia chartae animalis mortui creditur). Cette idée sera reprise par plusieurs auteurs, dont Antonio de Butrio dans son Tractatus de notorio art. I, $\mathrm{n}^{\circ} 11$, éd. Tractafus universi iuris IV, éd. Venise, 1584, et le Panormitain, sur X 2.22.10 n ${ }^{\circ} 13$; Cf. Lévy (1939, p. 102).

1) Cf. Pérez Martin (1981, 1982). 
Entre le XII ${ }^{c}$ et le $\mathrm{XIV}^{\mathrm{c}}$ siècle, deux modes de construction de la vérité se croisent. D'un côté, celui des preuves ordaliques, lié à une manifestation postulant la vérité comme pure assertion de culpabilité ou d'innocence. De l'autre, le système de la preuve qui révèle les diverses formes d'un modèle que l'on pourrait caractériser de la manière suivante. La vérité judiciaire produit une certitude probable qui n'est jamais indépendante du processus de sa reconstruction. Cette vérité, née comme un artifice, doit occuper le lieu indécis des faits, dans la mesure où -les juristes suivent en cela Aristote- il n'y a pas de certitude démonstrative à propos des événements. Il s'agit donc toujours d'une vérité dépendante de la restitution de la présence des faits absents afin de les rendre perceptibles, mais d'une vérité qui sait l'irréductible singularité et l'impossible répétition de ces mêmes faits. À partir du XV $\mathrm{XV}^{\mathrm{e}}$ siècle, un troisième mode de vérité s'élabore: celui qui tente de fixer le fait comme si le travail de reconstruction lui était étranger, comme si la vérité était celle d'un réel objectivement et entièrement récupérable. Un mode de construction de la vérité peut apparaître comme dominant à un moment ou à un autre, sans pour autant effacer entièrement le rôle joué par les autres.

Seconde observation. Dans la logique du système probatoire de la période classique construit par les glossateurs à partir d'éléments épars dans le droit justinien, le juge doit décider selon les preuves apportées. Mais jusqu'au XIVe siècle au moins, ces preuves, essentiellement testimoniales, sont l'objet d'une critique, et non pas simplement d'une application purement arithmétique. La conscience de l'erreur doit hanter le juge, et l'accumulation des fragments qui composent la vérité judiciaire doit être minutieuse et complète, d'une complétude qui est logiquement inatteignable, mais qui doit demeurer comme une tension nécessaire. Les textes offrent au juge différents procédés objectifs pour cela, même quand ils reprennent et glosent le texte du Digeste 22.5.3.2 qui affirme que l'on ne peut pas définir avec une totale certitude ce qui constitue une preuve suffisante.

Dans le même temps, le juge qui, comme dit saint Thomas, est une sorte de justice animée, peut se doter d'une objectivité qui est celle de la vérité de la Loi. Il ne fait que dire un droit qui le précède et l'efface comme sujet, accomplissant ainsi un geste qui donne à la représentation du juge comme sujet une autonomie objective. Par ce geste, le sujet s'affirme parce qu'il devient absolu, mais une fois ce geste fait, il efface toutes les traces de subjectivité qui risqueraient de l'affaiblir. Le juge est pris, comme la vérité qu'il énonce, entre un désir d'objectivité, d'adéquation au réel, et la subjectivité du geste critique, à la fois souveraine et récusée.

Mais un parcours qui, comme le mien, inclut à la fois les fors et le corpus alphonsin exige quelques justifications supplémentaires, puisque ces textes sont supposés appartenir à deux systèmes probatoires totalement différents. Je pense, pour ma part, que la réception du droit romain ne constitue pas le seuil au delà duquel tout bascule, mais bien plutôt le point de départ de changements progressifs, et que pendant le XIII ${ }^{\mathrm{e}}$ siècle s'affirment, au contraire, certaines formes probatoires qui étaient déjà centrales dans des textes des $\mathrm{XI}^{\mathrm{c}}$ et $\mathrm{XII}^{\mathrm{c}}$ siècles pas, ou peu influencés, par la renaissance romaniste.

Un des points majeurs de continuité entre les fors et les textes alphonsins est la primauté donnée aux témoins, à ceux qui ont vu et entendu. Perdure également l'idée selon laquelle témoigner est un honneur, qu'en être interdit est une punition infamante, et que la validité de ce qu'on dit est toujours fonction de l'identité sociale, qui compte plus que la cohérence interne de l'énoncé. Il y a, certes, entre les fors et les textes alphonsins une distance discursive et de méthode, un changement 
dans certains rituels et dans le déroulement des instances probatoires. Mais dans les textes alphonsins, en particulier dans ces deux textes qui eurent au XIII ${ }^{\mathrm{e}}$ siècle un destin théorique, l'Espéculo et les Siete Partidas, est formulé un cadre à l'intérieur duquel beaucoup d'opérations continuent d'exister, mais formalisées autrement. Le procès est, dans les fors aussi bien que dans l'œuvre du roi Sage, une sorte de jeu d'écrans, de dédoublements et recoupements de divers ordres, sociaux, logiques, narratifs, cognitifs. La preuve accueille, à la fois, des fictions et des éclats du réel.

Dans le cas des témoins, on peut distinguer dans les textes castillans du XIII siècle cinq critères majeurs de validation et de vérification dans la construction de la vérité. C'est à l'intérieur de ces critères, qui nous permettent de parcourir le chemin qui va de possibilités de la connaissance à la validation des récits, que s'inscrivent les acceptations et les exclusions du témoignage féminin. Un premier critère est celui de la perception sensorielle et la préférence pour les témoins de visu. Mais, deuxièmement, cette préférence est fortement limitée par l'assignation identitaire et la légitimité des déclarations en fonction de la personne du déclarant. Troisièmement, l'idée des connaissances fondées sur le mode de vie, ou sur les savoirs pratiques, justifie que les femmes puissent déposer sur les questions de virginité, grossesse et viol. Un quatrième critère est celui des différences et similitudes entre savoir et croyance. Et, finalement, on doit tenir compte de la logique interne des discours et de la confrontation des témoignages.

De là, le parcours qui inscrit les particularités propres à la procédure judiciaire castillane du XIII ${ }^{\mathrm{e}}$ siècle dans une analyse des concepts, des catégories et des représentations qui, dans l'Occident, fondent les critères de la preuve et les modes de certification.

1.- Le premier critère concerne donc entièrement la possibilité de la connaissance, les certitudes et les erreurs de la vision. La préférence du moyen-âge pour la voix vive des témoins comme forme de preuve s'appuie sur la primauté donnée par Aristote aux sens dans les processus cognitifs. Les grands commentateurs du XIV siècle reprennent la maxime scolastique - qui rend compte des Secondes analytiques (I, 18, 81a 38) dans lesquelles Aristote établit la nécessité de la sensation pour l'induction et la démonstration: "Nihil est in intellectus quod non prius fuerit in sensu $\gg^{12}$.

Dans le cadre de cette primauté sensorielle, la vue occupe la première place. La vision directe est censée transmettre une certitude cognitive. Avant les débuts du $\mathrm{XIV}^{\mathrm{e}}$ siècle, on ne rencontre pas de développement sur l'idée que les sens peuvent nous tromper. Pierre d'Auriol semble avoir été le premier à en faire un thème de réflexion. Mais les tromperies du savoir acquis par les sens dépendent, d'une part, de la complexité du processus sensible qui ne s'achève finalement que dans l'intellect, et d'autre part, des problèmes liés à tout ce qui ne relève pas de la vision directe: seule celle-ci réunit en effet les conditions de la connaissance vraie.

Dans le processus sensible, avec la seule perception visuelle, on ne sait encore rien sur ce que l'on voit en dehors des couleurs et des lumières disposées selon un certain ordre. La vision consiste dans la réception d'une forme qui se fixe d'abord dans la surface externe du cristallin, puis qui est transmise au corps subtil, qui se trouve dans la concavité du nerf, pour passer ensuite à la partie commune du nerf (le

12 «Rien n'est dans l'intellect qui n'ait d'abord été dans la sensation» Bartole, Tractatus de testibus, n. 4 (éd. Cologne, 1596). 
chiasma). Mais on ne sait rien encore tant que ce que l'on voit n'est pas saisi par d'autres sens que la vue. Des sensibles communs s'ajoutent en effet à ce que chaque sens fournit en propre; et leur appréciation ne peut s'opérer qu'au niveau d'un «sens commun " qui les compare, les sélectionne et les juge ${ }^{13}$.

Cette phase ultime de l'interprétation est commune aussi bien à la théorie qui postule que les rayons lumineux pénétrant dans l'œil, et qui a pour source la traduction faite au XIII ${ }^{\mathrm{c}}$ siècle de l'œuvre d'Alhazen, qu'à celles, défendues avec des variantes par l'optique antique et reprises pendant le moyen-âge, qui postulent l'existence de rayons visuels qui émanent de l'œil ${ }^{14}$. Adelard de Bath ( $\left.1116-1142\right)$, qui ignore l'œuvre d'Alhazen, dit dans ses Quaestiones naturales que de l'œil part un spiritum qui fait pression sur l'air en concurrence avec les formes des choses qui doivent être vues et que, façonné par cette rencontre, il revient à l'œil pour être soumis au jugement de l'anima ${ }^{15}$.

Mais à la complexité du processus de jugement et d'évaluation de la chose perçue, au cours duquel l'erreur peut survenir, s'ajoutent les problèmes liés à la réflexion et la réfraction. Aussi bien dans Ptolomée que dans les sommes monumentales de Alhazen et Vitellion (un moine polonais qui a traduit une partie de l'œuvre d'Alhazen au XIII' siècle), l'étude des images réfléchies et réfractées s'incrit toujours dans le cadre des erreurs visuelles et des illusions optiques. Reflexion et réfraction sont des leurres qui conduisent l'esprit à se méprendre sur le réel ${ }^{16}$.

Un texte rend compte de ces méprises de la perception en expliquant certaines illusions optiques et en recourant à des métaphores qui manifestent comment ce que l'on voit est marqué par ce que l'on est. Dans son De naturis rerum, Alexander Neckham (1157-1217) ${ }^{17}$ parle d'une série de phénomènes visuels qu'il qualifie de «dignes d'admiration » ou «merveilleux». Le soleil - dit-il - est comme le pouvoir, qui semble d'autant plus grand que l'on est plus éloigné de lui. Plus on est loin d'un homme puissant, plus on le considère digne de louange, mais si on obtient son amitié, on cesse de la désirer. Neckham utilise ensuite cette même image du pouvoir qui aveugle pour expliquer pourquoi, bien que le soleil soit rond, il ne semble pas avoir une surface convexe. L'impuissance de la vue et le pouvoir excessif du soleil l'expliquent, de la même façon que le lieu qu'occupent les hommes importants n'apparaît pas tel qu'il est en réalité, parce que leur apparente splendeur empêche de voir qu'ils sont, comme les autres hommes, soumis aux vicissitudes de la vie. Un homme, debout dans l'obscurité, voit celui qui est dans la lumière, mais celui-ci ne peut le voir, tout comme les gens d'obscure fortune perçoivent les actions des gens importants, alors que l'inverse n'est pas vrai. Un denier placé dans un bassin plein d'eau sera vu par quelqu'un qui se trouve loin du bassin, mais si on enlève l'eau, il ne le verra plus. C'est ainsi que quand l'âme est troublée, on observe des choses que l'on est incapable de percevoir quand elle ne l'est plus, et si beaucoup de prospérité

13 Simon (1981, II, p. 318-319).

14 Sur l'optique dans l'Antiquité, voir Simon (1988).

is Balossi, di Giovanni, Ferrari (1965, p. 53). Cette description coinncide avec ce que Aristote et Théophraste disent à propos de la thérie émanatiste de Démocrite, cf. Simon, (1981, p. 37). Adelard de Bath mentionne également ce qui semble être la version émanatiste d'Épicure qui, dans sa Lettre à Herodote, affirme que les atomes se détachent de la surface des choses en fines pellicules pour pénétrer l'œil (ibid., p. 53).

16 Simon (1988, p. 84).

17 Alexandri Neckham, De naturis rerum libri duo, éd. de T. Wright, Londres, 1863, p. 234-236. 
aveugle l'esprit, les inquiétudes donnent toute leur acuité à la vue. La colère agit en sens contraire: de même qu'on ne peut pas former une image en eau trouble, de même un esprit perturbé n'est pas attentif et est incapable de discerner la vérité. Les deux métaphores centrales employées par Neckham sont donc celle de l'aveuglement face au pouvoir, et celle des effets des troubles de l'âme. Si les difficultés de la vie aiguisent les sens, les états internes de perturbation, comme la colère, obscurcissent la perception. L'usage qu'il fait des métaphores manifeste que la compréhension des données sensibles n'est pas un phénomène stable mais qu'il est, tout au contraire, entièrement determiné par l'identité et les états de l'âme.

Au XIV siècle, Balde affirme dans sa glose au Décrétales de Grégoire IX que la vérité étant comme la lumière ${ }^{18}$, la connaissance visuelle est parfaite quand aucun nuage ne s'interpose entre elle et nos yeux. Mais en dehors de cette situation, à laquelle on peut seulement assimiler le notorium facti, l'evidentia rei qui est, par extraordinaire, constatée visuellement par le peuple et le juge, on ne perçoit la lumière que de manière indirecte, donc avec des risques d'erreur.

La complexité de la perception et de la vision indirecte déterminent des formes instables de la vérité transmise par ce sens, même quand on part de l'idée qu'il est premier dans la connaissance. Tant la complexité des processus visuels compris comme des processus interpretatifs, que les illusions produites par tout ce qui ne coïncide pas avec la vision directe, lorsque rien ne s'interpose entre l'objet et le regard, peuvent faire vaciller la certitude, ou l'identité parfaite de plusieurs perceptions. Avoir vu n'est donc jamais en fin de compte une preuve ex se. Ce n'est que par la légitimité identitaire qui autorise les paroles de ceux qui ont une bouche digne d'être crue, ou par la production grâce à la torture d'un témoin qui ne saurait mentir, que l'on achève de valider les récits des sens. La connaissance sensorielle des femmes se trouve ainsi doublement déterminée, par la complexité des processus cognitifs, qui concernent tous les êtres humains, et par le lieu social qui leur est propre.

2. - Afin de reconstruire un fait, de valider les narrations de ce que les témoins ont vu, le juge doit fixer les identités qui détermineront la «force» - le mot est employé par le Fuero Real à propos de l'estimation du témoignage des femmes -, l'indice de vérité qu'il peut attribuer à chaque énoncé. Non seulement les fors et le droit alphonsin, mais également le droit commun en général et la tradition scripturaire, rejettent certains témoignages en fonction de l'identité des témoins. Le critère des fors est en général celui de la «vecindad», qui implique la résidence dans une ville pendant une durée d'au moins un an et un jour en ayant «casa poblada», un patrimoine suffisant et l'exemption de certaines «pechas». Les femmes n'apparaissent pas fréquemment dans les références au témoignage - on parle bien souvent de «omnes vezinos»-, et, dans certains cas, quand on en parle, la mention tend à les exclure ${ }^{19}$.

Dans les Partidas, la liste des exclus du témoignage énumère les infâmes, le faux témoin ou toute forme de faussaire, celui qui cache la vérité par intérêt, ceux qui donnent des herbes pour tuer, pour avorter ou faire un mal physique à quelqu'un, les meurtriers (sauf ceux qui auraient tué en état de légitime défense), ceux qui ont des concubines étant déjà mariés, les violeurs et ceux qui auraient enlevé une femme ou

18 Sur X 2.19 rubr. «Veritas luci comparatur».

19 Par exemple dans le for de Soria, loi 277, éd. Sanchez (1919). 
séduit une religieuse, ceux qui seraient en situation de désobéissance par rapport à l'autorité dont ils dépendent (père, évêque, abbé), les incestueux, les traîtres et les alevosos, ceux qui «valent moins» ne pouvant être pairs d'un autre, les fous, les hommes de mauvaise vie comme les voleurs, les entremetteurs, les joueurs, les femmes qui s'habillent en homme, les hommes qui s'habillent en femme (selon l'Espéculo et le Fuero Real), ceux qui sont pauvres et «vils» et qui usent de mauvaises compagnies, ceux qui ont brisé leur hommage, les juifs et les maures dans un procès entre chrétiens, ceux qui ont moins de vingt ans, les femmes dans les testaments, les pères, mères, maris, femmes, parents jusqu'au quatrième degré, les esclaves libérés par ceux qui sont en cause, les dépendants de l'une des parties, les serfs (à moins de les torturer), et les ennemis ${ }^{20}$. Certaines catégories sont donc indépendantes de la conduite, alors que d'autres empêchements résultent des liens d'affection, de haine, ou de dépendance, et surtout, des actions des hommes que les textes alphonsins regroupent autour des idées de mala fama, d'erreur et de péché.

Le juge doit ainsi, en premier lieu, savoir qui parle, mais sans que son évaluation soit pour autant véritablement subjective. Les textes offrent les différents critères qui lui permettront de peser les témoignages comme s'il ne décidait pas -dans le sens fort de la décision. Tout comme pour la vérité du fait absent, les textes transmettent la conscience de l'impossibilité de saisir, sans lacune, la complexité du réel. Les critères d'évaluation des hommes n'ont jamais les conditions d'une pleine objectivité, ${ }^{21}$ bien que l'idée d'une permanence de la conduite permette de décider de l'avenir en fonction de ce que l'on sait du passé parce que «consuetudo quasi altera quaedam natura ${ }^{22}$.

L'Espéculo IV.7.32 justifie sa préférence pour les témoins masculins par le fait qu'ils ont «l'entendement plus certain et plus ferme ${ }^{23}$ que les femmes. Il serait vain de reprendre ici l'analyse d'un thème déjà suffisamment bien connu. Ce type d'argument est parfaitement courant, aussi bien dans le droit qu'ailleurs. Il me semble plus important de signaler que l'exclusion des femmes de la preuve testimoniale n'est que l'une des cristallisations identitaires qui permettent de fixer la reconstruction légitime d'un fait. En la comparant aux autres, on s'aperçoit même qu'elle est la plus variable. La pauvreté est un critère qui doit toujours être combiné avec les irrégularités de la conduite. L'exclusion des serfs qui ne seraient pas soumis à la question est stable et les exceptions sont régulièrement les mêmes. La minorité est fixée dans chaque texte sans que les arguments varient, même si l'âge le fait (entre quatorze et vingt ans). Mais en ce qui concerne le témoignage des femmes, les différences que l'on observe dans les textes du XIII ${ }^{c}$ siècle sont importantes et répondent à deux modèles différents de la connaissance. Le premier évalue leur capacité à percevoir, comprendre et reconstruire un fait dans un ordre purement quantitatif, leur connaissance pèse moins que celle des hommes, elle doit être «moins crue». Le second renvoie à un type de savoir d'ordre pratique, comme dans les constats de virginité et de grossesse, ou contextuel, lié à des formes spécifiques de sociabilité, à des lieux et des événements qui leur sont propres. Dans ce dernier cas, l'adéquation

20 Cette énumération est assez proche de celle que donne la Rhetorica Ecclesiastica, Wahrmund (1906, p. 71-73).

22 Cicéron, De finibus, V.74. Sur la nature comme «institution» dans le droit romain classique, voir le remarquable article de Thomas (1991).

23 «El sesso mas cierto et mas firme». 
entre sujets et énoncés fonde la vraisemblance du récit et la possibilité «féminine » de la connaissance.

La fixation d'identité qui filtre le récit brut en assignant des degrés variables de croyance en fonction du locuteur dépend, à l'évidence, d'une cohérence hiérarchique. Mais ce geste ne se réduit pas à une pure construction idéologique de la vérité. Un fait peut être perçu par les sens de n'importe quel individu - cela est fort bien prouvé par l'acceptation de n'importe quel témoignage quand il s'agit de sauver le roi. Mais la connaissance est différente selon la condition: aucun corps ne peut se soustraire à l'identité sociale qu'il pense posséder et, moins encore, à celle qu'on lui assigne. Ces déterminations, que le droit tente de fixer comme globalement stables, sont un écran cognitif et narratif dans la reconstruction de la perception sensorielle, non pas parce qu'elles jettent toujours de l'ombre, mais parce qu'elles éclairent diversement la perception du fait passé.

3. - Certains fors, le Fuero Real et les Leyes del Estilo coinncident sur le fait que l'on ne doit accepter les témoignages féminins que dans les litiges de femmes ou dans ceux où une femme au moins est directement impliquée. Cette décision tient compte du contexte du fait à prouver. Quand l'un des manuscrits du Fuero Real résume en tête le contenu de la loi où il est question de l'acceptation des témoignages des femmes, il mentionne les «lieux » qui leur donnent pertinence (En quels lieux peuvent témoigner les femmes) ${ }^{24}$. Il fait donc référence à certains lieux fixes, comme le four ou le lavoir, mais également à des situations de sociabilité strictement féminines, ou bien à celles dans lesquelles les femmes ont un rôle nécessaire et prépondérant. Dans certaines situations, elles peuvent faire une déposition dont la pertinence relève de l'intuition du sens, ou de leur savoir spontané quant à certaines pratiques et échanges sociaux, ce qui qualifie leur récit de manière positive dans le processus de reconstruction du fait. Alors que leur perception est souvent disqualifiée comme diffuse, ce qui limite normalement -sauf dans les Partidas- la possible vraisemblance de leurs récits, on peut se fier à elles quand elles parlent de situations que la société considère comme des expériences spécifiquement féminines. Leurs savoirs du corps, si important dans la communauté, se trouvent légitimés aussi dans l'espace judiciaire.

Dans les Siete Partidas 3.16.22 on fait mention de deux exceptions au serment qui précède le témoignage, et la seconde concerne la déposition des femmes sur des questions de grossesse: "S'il y a contentieux en raison d'un bien appartenant au mari décédé, dont la possession est demandée par l'épouse, parce qu'elle est enceinte de lui, et le juge ayant ordonné à des femmes expertes qu'elles l'examinent afin de savoir si elle est enceinte ou non, et celles-ci ayant dit au juge ce qu'elles pensaient : ces femmes-là ne doivent pas prêter serment, il suffit qu'elles disent simplement ce qu'elles pensent, si elle est enceinte ou non: et malgré que ces femmes disent leur témoignage par croyance, il doit être valable pour une question telle que celle-ci ${ }^{25}$. Lopez explique qu'il y a bien serment, mais serment de credulitate.

24 «En que lugares pueden testiguar las mugeres». Ms. P, de Perelada, Gerone, Biblioteca del Palacio, ms. 14.984, de la seconde moitié du $\mathrm{XV}^{\mathrm{t}}$ siècle.

25 «Si fuesse contienda en razon de alguna cosa que demandasse la muger, que la apoderassen de los bienes del marido finado, porque fincara prenada del; e mandasse el Judgador a algunas mugeres sabidoras, que la fuessen catar si era prenada, o non, e dixessen despues al Juez aquello que entendiessen: afales mugeres como estas non han por que jurar, mas abonda que digan llanamente 
Celui qui transmet ce que l'on a dans son cœur, et non dans sa mémoire, et qui suit le parcours augustinien de la vérité qui réside dans l'identité entre le sentiment de l'âme et les mots du discours. La croyance des sages femmes supplée les imperfections de la connaissance sensible quand des doutes se manifestent à l'égard du pouvoir cognitif de la vision et du toucher.

Deux chapitres des Décrétales parlent de la preuve de la virginité. Le premier pour dire que l'on doit croire plutôt l'épouse qui prouve sa virginité par l'inspection des sages-femmes que le serment du mari qui affirme l'avoir connue ${ }^{26}$. Le second ${ }^{27}$ pour penser la possibilité de l'erreur dans de telles expertises parce que, comme dit le canon, «souvent la main et l'œil des sages se femmes se trompent ${ }^{28}$. Balde donne, à propos de ce second chapitre (X 2.19.14), deux exemples des difficultés de la vision à percevoir le vrai qui illustrent, en réalité, le problème théorique de la difficulté à donner un témoignage à propos des phénomènes naturels dont la perception échappe aux sens: on peut voir pousser une calebasse sans en voir les atomes, même quand on la regarderait toute la journée, on ne perçoit pas de manière soutenue le mouvement de l'eau qui court en raison de sa vitesse, et il en va de même avec l'éclair. Face aux incertitudes de la connaissance sensible, les sages-femmes ne peuvent jurer que de credulitate, ce qui leur permettra, en cas d'erreur de bonne foi, d'échapper au parjure. Leur expérience ne se traduit pas dans un modèle cognitif de pleine certitude, même si dans la perspective aristotélicienne la mémoire, accumulant une multitude de souvenirs de la même chose «arrive à constituer finalement une seule expérience», et que d'elle naît l'art quand il "se dégage un seul jugement universel, applicable à tous les cas semblables " ${ }^{29}$.

Il y a donc les témoins qui ont vu, et qui disent ce qu'ils savent, et ceux qui disent ce qu'ils croient et qui dans certains cas, comme celui des sages-femmes, déposent sur ce qui, tout en relevant de leur perception sensorielle, dépend en fait d'un savoir pratique qui est la condition de la perception. Accepter l'existence de savoirs spécifiques, fragmentaires et non systématiques, implique l'acceptation de la relativité tant de la notion de «fait» que des fondements, instables et mouvants, d'une vérité nécessairement plurielle. Si le savoir est lié à la vision, la croyance se situe du côté des connaissances conjecturales. Aussi bien l'un que l'autre sont des rapports possibles entre le fait et l'évidence, et le premier ne produit pas plus de transparence du réel que la seconde.

4. - La notion de croyance est au centre de la construction judiciaire. Les textes reviennent constamment sur les manières de fixer qui doit être $c r u$, ou plus cru que les autres. L'Espéculo IV.7.30 a pour titre: «Quels témoins doit davantage croire le juge quand il y a désaccord entre leurs dépositions ${ }^{30}$, et le texte de la loi accumule une série de critères de croyance qui s'appuient d'abord sur la qualité et la quantité des témoins, puis sur la vraisemblance du récit qui permettent de construire la vérité comme le résultat de l'accumulation de fragments, de traces de la réalité.

aquello que entendieren, si es prenada, o non: e maguer tales mugeres digan su testimonio por creencia, deue valer sobre tal razon como esta...».

$26 \times 2.19 .4$.

$27 \times 2.19 .14$

28 «Saepe manus fallitur et oculus obstetricum», Décret de Gratien, C XXVII, q.1.c.4.

29 Aristote, Métaphysique, A.I (trad. de J. Tricot, Paris, 1966, 3-5).

30 «A quales testigos deue el juez mas creer quando acaesciere dessacuerdo en ssus dichos». 
Mais cette croyance qui construit par accumulation la vérité est multiple, aussi bien dans ses fondements que dans les degrés de conviction qu'elle transmet. Tous les textes du roi Sage interdisent la pratique des cojureurs, courante comme preuve dans les fors - surtout pour le défendeur dont la culpabilité n'aurait pas été prouvée par des témoins visuels. Ceux qui juraient ainsi avec l'accusé ne faisaient qu'attester sa valeur morale, l'impossibilité toute abstraite qu'il ait commis le fait dont on l'accusait. Et, contrairement aux témoins du droit romain qui devaient être, dans la mesure du possible, sans liens d'affection ou de dépendance avec les parties, les cojureurs étaient des voisins, ou des alliés de celui qu'ils appuyaient par leur serment. Les cojureurs se situaient ainsi d'emblée dans une croyance entièrement indépendante du fait. L'Espéculo et les Partidas exigent, au contraire, une croyance qui doit toujours être en rapport avec la mémoire, la connaissance du fait -ou du moins d'un fait qui puisse servir comme indice inférentiel. La première condition mise par l'Espéculo IV.11.29 pour éviter le parjure est que «l'on sache certainement, ou que l'on croit que c'est ainsi qu'il le jure. Et ce savoir on dit qu'il doit être quand on a vu ou on est sûr de quelque chose. Et la croyance doit être quand on a des indices, ou quand on a des raisons de croire la chose que l'on a entendue, malgré qu'on ne l'ai pas vue»"3!.

Les Siete Partidas 3.16.29, à la fin de l'énumeration des cas où le témoignage de auditu est accepté, concluent en disant que la déposition de celui «qui ne dit pas comment il sait ce sur quoi il témoigne, autrement qu'en affirmant qu'il le croit, son témoignage ne doit pas être tenu pour valable ${ }^{32}$. La glose de Lopez cite en premier lieu Saint Thomas en ce qui concerne la fides comme lieu intermédiaire entre l'opinio et la scientia, et Jean André qui distingue entre la croyance proxima, c'est-à-dire liée à la perception sensorielle qui permet d'atteindre la vérité, et celle separata qui ne fait pas preuve. La croyance ne doit pas être, suivant ces textes, entièrement disqualifiée. Si un témoin dépose à propos de ce qu'il a appris par les sens, il peut dire ensuite qu'il croit que c'est ainsi. Selon la glose de Lopez la croyance ne prouve pas, mais elle ratifie. Mais si croyance et savoir apparaissent chez le juriste impérial comme deux formes qui ne participent plus d'une même essence, au XIII siècle, c'est bien le processus d'accumulation de croyance qui conduit à la vérité judiciaire. Les Siete Partidas 3.11.11 avertissent celui qui s'apprête à jurer d' «asmar en su coraçon $»^{33}$ s'il croit sans douter. Mais la glose de Lopez n'accepte plus la réponse qui utilise le verbe croire. On ne doit qu' affirmer, ou bien nier, comme si la croyance rendait douteuse l'affirmation.

Â la fin du $X V^{e}$ siècle, la fides ou credulitas qui donne accès aux vérités probables, les seules que la preuve classique croit pouvoir établir, est devenue insuffisante pour la construction logique et rituelle de la vérité judiciaire. À partir du XV siècle on reconnaît au monde des « faits " une autonomie objective qui efface l'écart par rapport au réel dont la preuve classique était consciente et qui la renvoyait constamment aux vérités probables et à l'assensum ${ }^{34}$. La notion même de fides avait commencé, chez les canonistes, au XIII ${ }^{\mathrm{e}}$ siècle, un processus de dépréciation inau-

31 «Ssepa bien ciertamjente, o cree, que assi es lo que jura o non es assy. Et este ssaber dezimos que deua sseer, veyendo lo o acertando sse en ello. Et el creer otrossy deue sseer, aujendo ende tales ssenales o sseyendo la cosa que oyere tan con rrazon por que aya de creer, maguer non la vea».

32. «Que non diere razon de como sabe lo que testigua, si non que dize que lo cree, que non deue valer aquello que atestiguare».

33 D'évaluer, de penser dans son cour.

34 Giuliani (1961, p. 208). 
guré par Henry de Suse dans sa Summa aux Décrétales de Grégoire IX, qui la transformait dans un nomen non finitae qualitatis selon le schéma doctrinal du nomen infinitum. Reléguée dans l'accidentalité et la fausseté cognitive, la fides justifiait l'intervention du magistère de l'Église afin d'organiser la connaissance de l'homme, perdu dans les mille prédicats possible d'une foi sujette à tous les accidents ${ }^{35}$.

Mais au XIII ${ }^{c}$ siècle la croyance est encore une notion riche en modulations, en potentialités cognitives diverses qui fondent, selon une échelle variable, le récit de ceux qui parlent et l'adhésion de ceux qui écoutent.

5. - Le dernier critère de validation est celui du rapport que chaque récit entretient avec la vérité du point de vue de la narration, la manière dont est reconstruite une réalité effacée mais dont les contours peuvent être restitués par la voix des témoins, les pièces écrites, les indices, et la présomption qui, tout en étant relative en tant que preuve, constitue le modèle même de la connaissance juridique du système classique parce que, comme lui, elle relève du doute et du probable.

La définition de la preuve comme «rei dubiae per argumenta facta demonstratio » est fort courante dans le droit commun. Elle contient deux éléments essentiels. D'une part, celui qui installe le doute au cœur de la preuve, parce qu'elle relève toujours de ce «rei dubiae». Cette partie de la définition n'est pas contestée au XIII siècle, elle ne le sera que vers la fin du siècle suivant, dans l'œuvre de Balde qui défend une notion de preuve dont la nature n'est pas nécessairement liée au doute ${ }^{36}$. Le second élément, l'usage du mot argumenta, est au contraire plus problématique puisque, pour certains, il limite la preuve à ce que la Rhétorique d'Aristote nommait les «preuves artificielles», c'est-à-dire celles fournies par l'orateur, en dehors de tout témoignage, écrit ou serment, de toute trace du fait - qu'il apellait «preuves non-artificielles ${ }^{37}$. Geoffroy de Trani élimine prudemment toute référence aux argumenta en faveur d'une énumération qui inclut témoins, écrits, indices et présomptions ${ }^{38}$. Les textes alphonsins suivent cette dernière définition ${ }^{39}$ qui semble vouloir réduire l'emprise de la démonstration argumentative. Mais au delà des mots retenus, l'Espéculo et les Siete Partidas élaborent une vraisemblance qui est en

35 Montorzi (1984, p. 29-33). Nomen infinitum, c'est le nom sans référent ontologique, qui désigne des réalités négatives: nihil, nox, peccatum, silentium, non homo. C'est donc un nom qui est un pur prédicat; voir note 45 , p. 32 .

36 Sur $\mathrm{C} 4.19$ rubr. $\mathrm{n}^{\circ} 7$ Balde critique Nicolaus de Matarellis qui considère que la preuve est «extrinseca demonstratio rei dubie per modos a iure statutos «parce que l'idée d'une démonstration nécessairement extrinsèque est à ses yeux erronce. Et si Matarellis propose une division des moyens de preuve selon laquelle la certitude absolue du fait que l'on constate est au delà de la preuve comme son excès («Excedunt, complent, mediant, prosuntque probandi»), Balde met à la place de l'excès ce qu'il considère comme la meilleure des preuves: la "probatio cum certa certitudine».

37 Le mot argumentum est ambigu, puisque pour certains, il désigne aussi bien preuves artificielles que non-artificielles. Ainsi dans la Summa Codicis (éd. Fitting, Berlin, 1894) attribuée à Irnerius, IV.19.3 «Argumenta quidem sunt quae causam indicent atque ostendunt, et ideo testes et instrumenta item inditia sub hac appellatione continentur» [les argumenta sont, en effet, celles qui indiquent et démontrent la cause, les témoins et les écrits et aussi les indices sont donc contenus sous cette dénomination].

38 Geoffroy de Trani, dans sa Summa super titulis decretalium, éd. Venise, 1570, rubr. de probationibus, $\mathrm{n}^{\circ} 1$, propose «probatio est rei dubiae per testes et instrumenta et plerumque indicia et praesumptiones ostension [la preuve est la démonstration d'une chose douteuse au moyen de témoins et d'écrits et fréquemment au moyens d'indices et de présomptions].

39 Espéculo V.10.1 et Siete Partidas 3.14.1. L'énumération des moyens de preuve se trouve dans Siete Partidas 3.14 .8 où il n'est pas question d'argumenta. 
grande mesure dépendante de la logique du récit et qui repose sur trois fondements: la pertinence des preuves pour un factum probandum aux contours précis et limités, la confrontation des témoignages, et le sens de la praesumptio ( "sospecha»).

Tout d'abord, en ce qui concerne la pertinence des preuves par rapport au fait à prouver, on peut signaler que dans l'Espéculo IV.7.30, quand les deux parties présentent, en même temps, des témoins qui se contredisent, le juge doit suivre ceux qui sont plus honorables, ou de "meilleures vie ou coutumes», ou «ceux d'entre-eux dont les dires s'approchent plus de la vérité en disant des choses qui touchent plus spécifiquement le fait, et ils doivent juger selon cela ${ }^{40}$. Mais comment mettre comme une condition même de la procédure l'existence de ce que l'on essaye justement d'établir, à savoir, la vérité du fait? Il y a en réalité deux manières de circonscrire la pertinence des preuves apportées. D'une part, c'est dans la narratio et la responsio que se trouvent disséminées les traces de la vérité que le juge doit repérer. D'autre part, la définition d'un status - le fait à prouver - comme «centre d'argumentation » et critère de pertinence des preuves fait qu'il est possible de situer la vérité que l'on tente de construire, non dans l'idée d'un fait réel et purement objectif qu'il serait possible de restituer, mais dans celle du rapport entre le fait qui doit être prouvé, construit comme une quaestio, et les preuves qui vérifient la validité de son institution discursive. Le fait qui doit être prouvé est, à la fois, le produit d'un tri entre les traces du réel et d'une série de distinctions discursives.

Le deuxième critère de vraisemblance du récit réside dans la confrontation des témoignages. Elle s'organise dans l'Espéculo IV.7.19 selon cinq «choses» qui doivent coïncider. La première est la «chose» et le «fait»: l'exemple donné est celui des objets en jeu, qu'ils soient l'objet du litige (réclame-t-on une maison ou une vigne?) ou un objet qui entre de manière essentielle dans l'affaire (a-t-on été frappé avec une pierre ou un bâton?). La deuxième, c'est les personnes. La troisième concerne le degré de parenté quand il est pertinent. La quatrième est le lieu. Et la cinquième est le temps, où il faut néanmoins distinguer entre la chose qui ne peut arriver qu'une fois (la mort, le viol d'une vierge ou la perte d'un membre), pour laquelle il ne saurait y avoir de désaccord, et celle qui admet la répétition et pour laquelle la non-cö̈ncidence des témoignages ne conduit pas à leur invalidation (l'adultère, la fornication, la blessure, le vol, etc.).

Les Siete Partidas 3.16.28 ne font pas d'énumération systématique. Elles parlent du temps et du lieu, de la présence d'autres témoins du fait, et elles suggèrent que si le témoin est un homme honorable, le juge n'a pas à aller plus loin. C'est seulement s'il est face à un homme «vil » qu'il doit poser des questions portant sur le temps qu'il faisait, le type de vêtements que les gens portaient, et d'autres encore qui permettent de déceler le possible mensonge. À propos de cette loi, la glose de Lopez plaide, à l'aide de Saint Thomas, pour une coïncidence de sens qui ignore les détails du récit. La vérité ne doit pas être cherchée dans une cohérence obsessionnelle dont l'exactitude des moindres détails feraient la crédibilité. L'oubli de certaines choses est naturel aux hommes, qui n'essaient pas de tout retenir dans leur mémoire. Pour Saint Thomas, une parfaite coïncidence serait même, a contrario, une preuve de la fausseté de la déposition ${ }^{41}$.

Le troisième critère d'évaluation des récits des parties et des témoins est celui de la praesumptio. Celle-ci implique une idée de ce qui est normal, probable, qui sera

\footnotetext{
40 "Quales dellos sse acuestan mas ssus dichos a la verdat diziendo rrazon que tanga mas ssenaladamjente al ffecho; et ssegunt aquello deue judgam.
}

41 Il s'agit de la Summa theologiae, IIa, IIae, q. 70, art. 2. 
fondée, au moins jusqu'à la moitié du XIII ${ }^{e}$ siècle, dans une probabilité moralement orientée. C'est l'opinion commune qui détermine la probabilité, et non pas la fréquence d'un fait. Le rapport entre praesumptio et «id quod plerumque accidit $\aleph^{42}$, présente chez Aristote, sera effectivement liée à la diffusion de la Logica nova aristotélicienne, sans faire pour autant l'unanimite ${ }^{43}$. Dans les textes alphonsins en tout cas les formes de la praesumptio ne sont pas fondées sur la fréquence, mais sur les valeurs établies et reconnues comme nécessaires, jusqu'à preuve du contraire.

La praesumptio décontextualise et fixe la complexité des conduites en produisant une série de lieux communs qui déterminent la vraisemblance des récits (l'âge ne change pas la nature d'une personne, les hommes puissants ne peuvent avoir peur des faibles, un père ne voudrait pas déshériter un fils afin de tout donner à un autre, etc.). Quand l'acceptation d'un argument implique la reconnaissance d'une chose qui semble peu probable ou tout à fait absurde selon une normalité éthique, alors le juge doit douter qu'elle conserve un rapport à la vérité, à moins que l'on soit capable de la prouver, et c'est en cela que la praesumptio peut indiquer sur qui retombe le poids de la preuve.

Où se situe le récit des femmes à l'intérieur de ces critères qui conditionnent et manifestent toute reconstruction véridique des faits? Il faut dire que les fors ne font aucune place à la praesumptio, et que la détermination de la cause y est moins nette, plus massive. Mais sans en faire une théorie ou une forme probatoire, ces textes s'appuient très ostensiblement sur la probabilité moralement orientée des conduites humaines et, encore plus que les textes alphonsins, en particulier dans le cas des femmes, sur l'idée d'un savoir commun qui est matrice de la connaissance. Mais cette matrice, qui est loin d'être unique, s'articule sur les divers registres qui permettent de rendre compte de la diversité du réel.

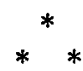

Tout indique que la notion de preuve rencontre des obstacles dans sa recherche de l'objectivité. Dans la mesure où il n'y a pas une objectivité absolue des moyens de preuve, et où ces moyens reconstruisent, avec distance, ce qui s'est passé, chacun des différentes aspects du témoignage renvoie comme dans un jeu de miroirs aux autres. Au delà des différences qui séparent les textes qui scandent la pensée juridique du XIII ${ }^{e}$ siècle castillan, tous retiennent, à des degrés divers, l'idée que la vraisemblance et, surtout, l'acceptation du témoignage, dépendent de l'identité du témoin, mais également de la logique argumentative et de la forme de la perception sensorielle. La vérité, qui est le produit d'une accumulation suffisante de croyance de la part du juge, dépend des contextes d'énonciation où chaque récit prend place, des différents «jeux du langage».

Alors que les fors et le Fuero Real désignaient explicitement des situations propres aux femmes, l'Espéculo leur attribue, en cas de procès criminel, une moindre contribution dans la construction d'une vérité pour laquelle elles ne peuvent offrir, indépendamment de leur certitude personnelle, que des indices qui permettent au juge de diriger son effort vers les mots d'un autre. Et les Partidas, qui plaident pour le seul critère de la bonne réputation, rappellent que dans les cas où le

42 «Ce qui arrive fréquemment ».

43 Giuliani (1961, p. 66 et sq.). 
témoignage des femmes est non seulement fréquent mais encore indispensable, elles ne jurent pas de dire la vérité - elles jurent de credulitate, dira la glose - même si leur connaissance relève de l'expérience sensible.

À la fin du XIII ${ }^{\mathrm{e}}$ siècle, les Leyes del Estilo reviendront sur une conception du savoir féminin qui était celle des fors : lieux, gestes et occasions vont établir la probabilité de leur véridicité, non parce que l'on croit qu'elles mentent, mais parce que ce n'est que là qu'elles savent.

Ainsi, le récit féminin se situe essentiellement dans l'espace des pratiques ou des expériences spécifiques qui fonctionnent comme des univers partiellement ou totalement fermés, opaques à la connaissance masculine. Seules les Partidas tentent de postuler une connaissance qui se situe au delà de l'identité féminine, même si dans des nombreux passages elles acceptent l'opinion commune selon laquelle leur esprit est plus faible que celui des hommes. Mais cette objectivité que les Partidas sont les seules à tenter d'instituer ne durera pas. En 1290, les Leyes del Estilo reviennent à l'idée que les femmes doivent déposer exclusivement dans des litiges de femmes, dans des situations de femmes.

Reste que dans tous les textes, à des degrés divers, le témoignage des femmes participe de l'accumulation qui permet d'unifier les narrations dans un récit que l'on donne pour vrai, parce qu'il est «probablement» vrai. Il n'y a pas de meilleure définition de ce geste que la deuxième proposition énoncée par Ludwig Wittgenstein dans De la certitude: «De ce qu'à moi, ou à tout le monde, il en semble ainsi, il ne s'ensuit pas qu'il en est ainsi. Mais ce que l'on peut fort bien se demander, c'est s'il $y$ a sens à en douter ${ }^{44}$.

Au terme de ce parcours, dans et hors la Castille médiévale, je voudrais faire retour à ma question initiale. Dans le XIII ${ }^{\mathrm{e}}$ siècle castillan, alors qu'aucune transformation fondamentale ne modifie les déterminations ou les manifestations de l'infériorité féminine, les pratiques judiciaires font succéder à un mouvement vers l'acceptation des témoignages féminins, selon les critères qui valent pour tout témoignage, un retour à une stricte limitation de la pertinence accordée aux paroles des femmes. La force maintenue des catégories qui étaient celles des fors, reprises et reformulées dans les Leyes del Estilo, l'emportent pendant tout le XIII ${ }^{\circ}$ siècle sur les propositions des Siete Partidas. C'est donc à l'intérieur des logiques propres aux procédures et aux opérations juridiques que l'on peut comprendre les raisons, les modalités et les conjonctures de l'exclusion féminine. Celle-ci a des formes diverses selon les différents domaines où elle est manifestée. L'histoire des femmes, en Espagne comme ailleurs, ne doit donc pas dissoudre ces différences et ces écarts dans une vision trop globale et trop linéaire de l'assujettissement féminin. Tout au contraire, cette histoire doit reconnaître les figures propres, les logiques spécifiques et les discours particuliers qui le designent et le formulent.

Marta MADERO

Universidad de Buenos Aires Universidad Nacional de General

Sarmiento

Université Paris XIII mmadero@impsat1.com.ar

44 Traduction de Fauve $(1965$, p. 31$)$. 


\section{RÉFÉRENCES}

Balossi, S., di Giovanni, A., Ferrari, B., (Dir.) «Quaestiones naturales» di Adelardo di Bath, Rapallo, 1965.

Giuliani, A., Il concetto di prova. Contributo alla logica giuridica, Milan, Giuffrè, 1961.

Lévy, J.-Ph., La hiérarchie des preuves dans le droit savant du moyen-âge, Annales de l'Université de Lyon, 1939, pp. 127-128.

Montorzi, M., Fides in rem publicam. Ambiguità e techniche del diritto comune, Naples, Jovene Editor, 1984.

Pérez Martin, A., El ordo iudiciarius «Ad summariam notitiam» y sus derivados. Contribución a la historia de la literatura procesal castellana, Historia, Instituciones, Documentos 1981,8 , p. 195-266, 1982, 9, p. 327-423.

Sanchez, G. (ed.), Los fueros castellanos de Soria y Alcalá de Henares, Madrid, 1919.

Simon, G., «Derrière le miroir», Le temps de la réflexion, 1981, (vol. 2).

Simon, G., Le regard, l'être et l'apparence dans l'optique de l'Antiquité, Paris, 1988.

Thomas, Y., «Imago naturae. Note sur l'institutionnalité de la nature à Rome ", in Théologie et droit dans la science politique de l'État moderne, Actes de la table ronde organisée par l'École Française de Rome et le CNRS, Rome, 12-14 novembre 1987, École Française de Rome, 1991, p. 201-227.

Wahrmund, L., (Ed.) Quellen zur Geschichte des römisch-kanonischen Processes im Mittelalter, Innsbrück, Heft IV, 1906.

Wittgenstein, L., De la certitude, traduction de Fauve, Paris, Gallimard, 1965. 